\title{
Gamidactylus bryconis sp. n. (COPEPODA, POECILOSTOMATOIDA, VAIGAMIDAE) DAS FOSSAS NASAIS DE PEIXES, Brycon pellegrini HOLLY, 1929 E B. melanopterus (COPE, 1872) DA AMAZÔNIA BRASILEIRA.
}

\section{Angela VARELLA'}

RESUMO - Gamidactylus bryconis sp. n. foi coletada nas fossas nasais de Brycon pellegrini $\mathrm{e}$ B. melanopterus. A nova espécie é semelhante a Gamidactylus jaraquensis, por possuir antena com espinhos móvel distal no terceiro segmento e garra terminal, além de um par de retroestiletes laterais móveis, no primeiro somito torácico. Difere da espécie já conhecida no comprimento e forma dos retroestiletes e na ornamentação das pernas.

Palavras-chave: Copepoda, Poecilostomatoida, Parasitas de Peixes, Amazônia, Brasil.

Gamidactylus bryconis sp. n. (Copepoda, Poecilostomatoida, Vaigamidae) from the nasal fossae of fishes, Brycon pellegrini HOLLY, 1929 e B. melanopterus (COPE, 1872) from the Brazilian Amazon.

ABSTRACT - Gamidactylus bryconis sp. $n$. was collected from the nasal fossae of Brycon pellegrini and B, melanopterus. The new species resembles Gamidactylus jaraquensis in having antennae with moveable spines distally on the third segment and terminal claws, and a pair of strong moveable lateral retrostylets on the first thoracic somite. The new species can be distinguished from its congeners in the size and shape of the retrostylets and in the armature of the legs.

Key-words: Copepoda, Poecilostomatoida, fish parasites, Amazon, Brazil.

\section{INTRODUÇÃO}

As fêmeas de copépodos ergasilóides são parasitas de peixes conhecidas em todo o mundo, por causarem severos danos, principalmente em cultivos intensivos. São frequentemente encontradas nos filamentos branquiais ou fossas nasais e mais raramente na pele e nas nadadeiras de seus hospedeiros (THATCHER, 1991; VARELLA, 1992).

A família Vaigamidae foi proposta por THATCHER \& ROBERTSON (1984), para abrigar os copépodos ergasilóides que possuem retroestiletes dorso-laterais móveis no primeiro somito torácico. Os autores propuseram o gênero Vaigamus como tipo para a família e descreveram macho e fêmea de duas espécies encontradas no plâncton, na Amazônia Central: Vaigamus retrobarbatus e $V$. spinicephalus. A partir daí, novas espécies de vaigamídeos têm sido coletadas nas fossas nasais de vários peixes amazônicos (Gamidactylus jaraquensis Thatcher \& Boeger, 1984; Gamispinus diabolicus Thatcher \& Boeger, 1984b e Gamispatulus schizodontis Thatcher \& Boeger, 1984).

A primeira espécie do gênero Gamidactylus, $G$. jaraquensis Thatcher \& Boeger, 1984, foi descrita das fossas

1 Coordenação de Pesquisas em Biologia Aquática, Instituto Nacional de Pesquisas da Amazônia, INPA, Caixa Postal 478, Manaus, Amazonas, Brasil, CEP 69.011-970. 
nasais de Semaprochilodus insignis (Schomburgk, 1841). Esta espécie também foi encontrada nas fossas nasais de Pygocentrus nattereri (KNER, 1860), juntamente com Rhinergasilus piranhus Boeger \& Thatcher, 1988.

Gamidactylus bryconis sp. n. é a segunda espécie do gênero descrita para a Amazônia.

\section{MATERIAL E MÉTODOS}

Os peixes utilizados neste trabalho foram coletados em duas excursões realizadas no Estado de Rondônia, a primeira de 28 de novembro a 8 de dezembro de 1984 e a segunda de 21 de setembro a 3 de outubro de 1985 . Estes peixes foram identificados, as fossas nasais removidas e fixadas em formal $10 \%$.

Os copépodos foram retirados das fossas nasais no laboratório. Lâminas permanentes, com montagem total dos organismos, foram preparadas usando o método Thatcher, denominado "HYP", descrito por SANTOS-SILVA, et al. (1989).

Os desenhos e medidas foram feitos com microscópio óptico provido de câmara clara e ocular micrométrica. As medidas estão expressas em micrômetros.

\section{RESULTADOS}

Vaigamidae Thatcher \& Robertson, 1984. Gamidactylus Thatcher \& Boeger, 1984. Gamidactylus bryconis sp. $\mathrm{n}$.

(Figs. 1 - 12)

Material examinado:

Holótipo: fêmea (INPA-CR 631), das fossas nasais de Brycon melanopterus, coletado no rio Jamari, próximo à represa de Samuel (8'45"S e 63'28"W), 07 de dezembro 1984, em lâmina. Parátipos: 15 fềmeas das fossas nasais de $B$. melanopterus e $B$. pellegrini (INPA-CR 632a - p) e 3 fêmeas (MZUSP 10448a-c) todos em lâminas; coletados no rio Jamari, próximo à represa de Samuel e no rio Mamoré, próximo à Surpresa . Coletados por MALTA, J. C. O.

Descrição da espécie: baseada em 18 exemplares estudados e 10 medidos (Tabs. 1 e 2). Cefalotórax (Fig. 1) ovalado anteriormente. Cabeça fusionada com o primeiro e segundo somitos torácicos. Olho no terço anterior do cefalotórax, com coloraçäo azul cobalto (cor 68, SMITHE, 1975) e coloraçäo do corpo dada por poucos pigmentos de tonalidade azul esmalte (cor 70, SMITHE, op. cit.). Retroestiletes (Figs. 1 e 2) longos e pontiagudos; projetando-se pósterolateralmente do primeiro somito torácico, atingindo a borda posterior do cefalotórax. Tórax (Fig. 1) com quatro somitos livres.

Somito genital duplo (Fig. 3) subretangular. Somito genital duplo, somitos abdominais 1 e 2 (Fig. 3), providos de fileira de espínulos ventrais que se estende lateralmente até a borda dorsal. Somito anal com acentuada incisão mediana $\mathrm{e}$ também provido de espínulos marginais. Ramos caudais (Figs. 1 e 4) com fileira externa de espínulos e duas setas terminais, a interna cerca de 2,5 vezes mais longa que a externa.

Antênula (Fig. 5) com seis segmentos semelhantes em tamanho, 


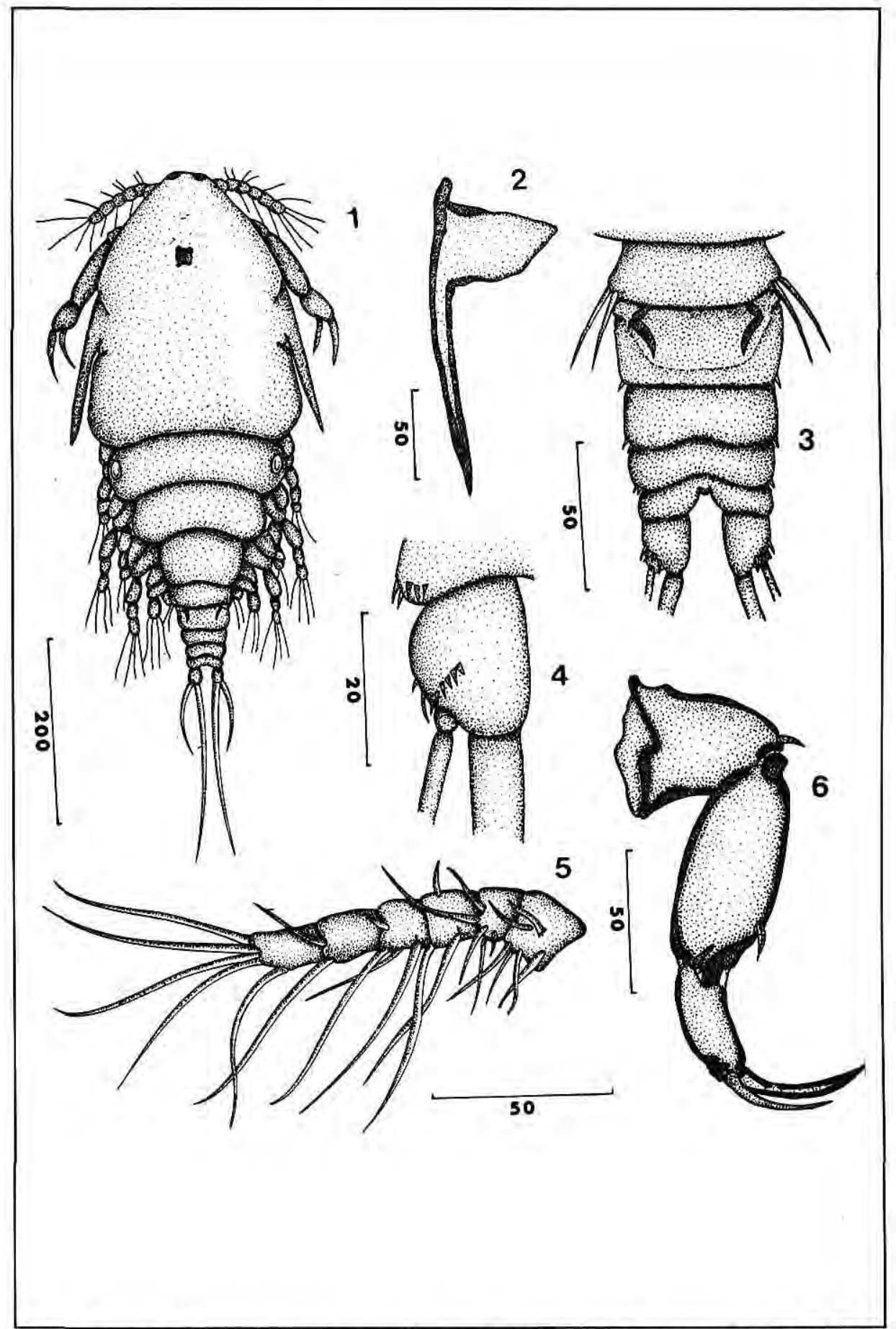

Figuras 1-6. Gamidactylus binconis sp. n. (lềmea). I - Vista dorsal; 2 - Retroestilete; 3 - Abdome, somito genital duplo e ramos caudais; 4 - Ramo caudal (vista ventral); 5 - Antênula; 6 - Antena. 


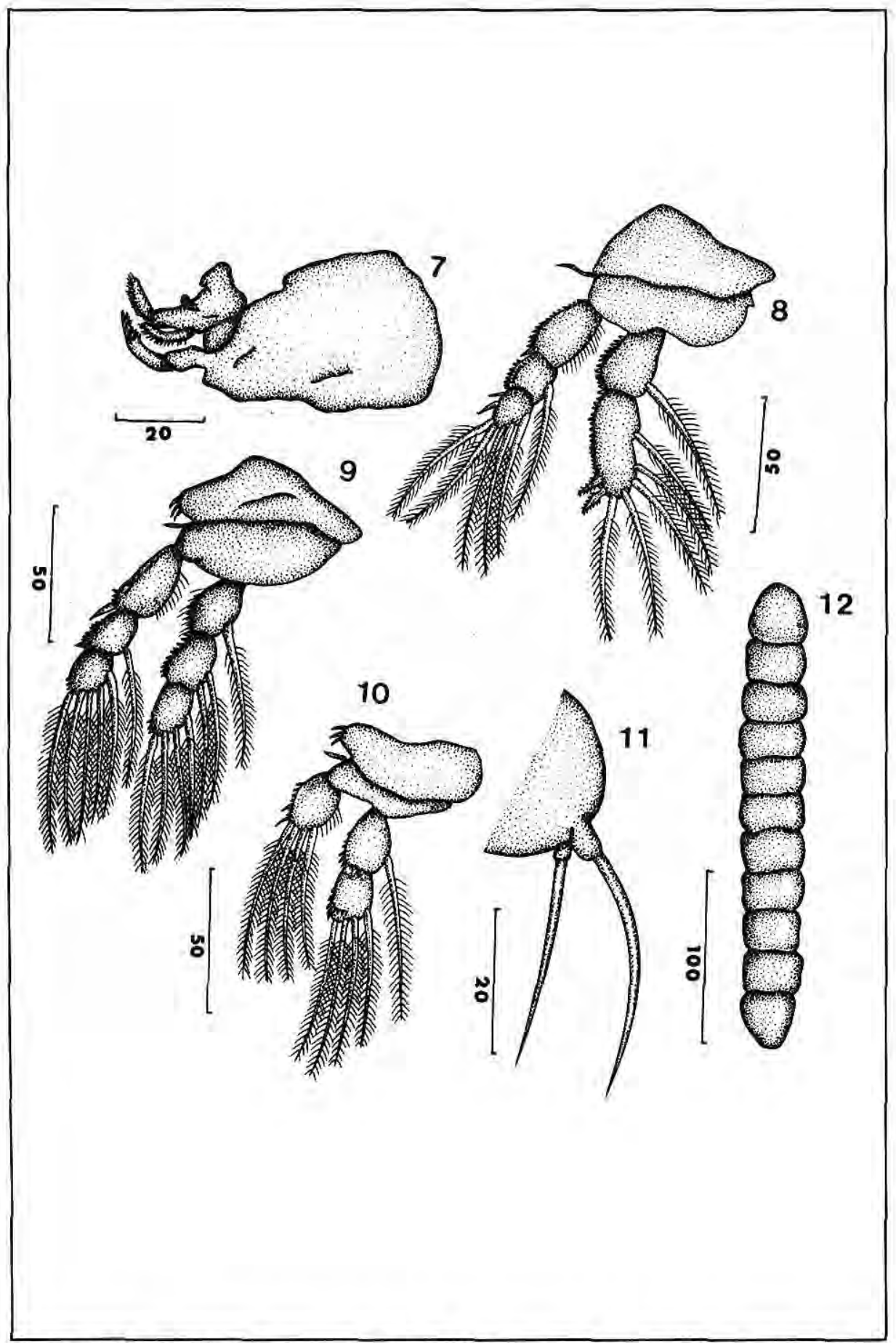

Figuras 7-12. Gamidactylus brycomis sp. $\mathrm{n}$. 7 - Peças bucais; 8 - Perna I; 9 - Perna II = III; 10 - Perna IV; II - Perna V; 12 - Saco ovígero. 
providos de 25 setas lisas (n. de setas/ segmento: $6-5-3-4-2-5$ ).

Antena (Fig. 6) com três segmentos e garra. Segmento 1 apresenta um espinho distal interno; segmento 2 com um espinho subterminal interno; segmento $3 \mathrm{com}$ um longo espinho distal, móvel; garra provida de poro proximal. A relação entre os segmentos é: 1,0:1,2:0,6:0,7(garra).

Peças bucais (Fig. 7). Mandíbula bi-segmentada, com segmento terminal provido de cerdas na margem posterior; palpo com cerdas. Maxílula reduzida. Maxíla bi-segmentada, com o segmento terminal curvo e provido de pequenos espinhos na extremidade distal.

Pernas (Figs. 8 - 11 e Tab. 3). Os quatro primeiros pares são natatórios e birremes. Coxopoditos das pernas II a IV com três espinhos na margem externa. Basipoditos das pernas I a IV com seta lateral externa lisa. Perna I (Fig. 8) com endopodito de dois segmentos, ambos com espínulos externos. O primeiro segmento do endopodito com seta plumosa na margem interna; o segundo com dois fortes espinhos pectinados distais e cinco setas plumosas. Exopodito com três segmentos. $\mathrm{O}$ primeiro segmento com espínulos externos, um espinho distal e cerdas internas; o segundo segmento com duas fileiras de espínulos externos e uma seta plumosa mediana interna; o terceiro segmento com dois espinhos e cinco setas plumosas. Pernas II e III (Fig. 9) semelhantes, com ambos os ramos constituídos por três segmentos. O endopodito com os dois primeiros segmentos providos de espínulos externos. O primeiro segmento com uma seta plumosa interna e cerdas externas; o segundo com duas setas plumosas internas; o terceiro com três espínulos externos e quatro setas plumosas. O primeiro segmento do exopodito com três espínulos externos e um espinho distal; o segundo com espínulos externos, um espinho distal e uma seta plumosa mediana interna; o terceiro com espínulos externos e cinco setas plumosas. Perna IV (Fig. 10) com o endopodito bi-segmentado e ambos os segmentos com espínulos externos, atingindo até a metade da margem distal. O primeiro segmento com uma seta plumosa mediana interna; o segundo com quatro setas plumosas. Exopodito com apenas um segmento provido de espínulos externos, dois espinhos e quatro setas plumosas e cerdas internas. Perna V (Fig. 11) reduzida a duas setas lisas cada uma articulada a uma papila lateral no sexto somito torácico.

Saco ovígero (Fig, 12) com uma média de 11 ovos em série única.

Macho desconhecido.

Etimologia: O nome específico deriva do nome genérico do hospedeiro, Brycon.

\section{DISCUSSÃO}

Gamidactylus bryconis asse-melhase a $G$. jaraquensis, mas possui as seguintes características diferenciais: I) retroestiletes mais fortes e retos, não ultrapassando a borda do cefalotórax; em G. jaraquensis estas projeções são mais delicadas e longas, atingindo o primeiro par de pernas; 2) primeiro segmento do exopodito das pernas I a III com um único espinho; $G$. jaraquensis 
apresenta, correspondentemente, dois espinhos; 3) a relaçäo entre o comprimento da garra e o terceiro segmento da antena é maior para $G$. jaraquensis que para $G$. bryconis; 4) em $G$. bryconis os basipoditos II a IV possuem setas lisas, enquanto que em $G$. jaraquensis estes artículos não apresentam ornamentações; 5) os ramos caudais de $G$. bryconis são mais robusto que em $G$. jaraquensis.

O local de implantaçäo dos retroestiletes no cefalotórax varia nas duas espécies: em $G$. jaraquensis a inserção se dá na porção média e em $G$. bryconis é mais anterior, dando a impressão, que este órgão é maior em $G$. jaraquensis do que em G. bryconis, porém, comparando-se suas médias de comprimento, respectivamente 131 e 171, verifica-se que os retroestiletes de $G$. bryconis são maiores que os de $G$. jaraquensis.

Apesar de $G$, jaraquensis não apresentar uma grande especificidade em relaçäo aos seus hospedeiros, as características enumeradas indicam que $G$. bryconis trata-se de uma espécie distinta,

Tabela 1. Medidas $(\mu \mathrm{m})$ de 10 fêmeas adultas de Gamidactylus bryconis sp. $\mathrm{n}$.

\begin{tabular}{|c|c|c|}
\hline & COMPRIMENTO & LARGURA \\
\hline Corpo (sem setas caudais) & $464-576(535)$ & $162-220(201)$ \\
\hline Cefalotórax & $232-288(271)$ & $162-220(201)$ \\
\hline Retroestilete & $146-182(171)$ & $\cdots$ \\
\hline \multicolumn{3}{|l|}{ Somitos torácicos livres } \\
\hline 111 & $36-68(52)$ & $136-172(159)$ \\
\hline IV & $42-58(54)$ & $110-136(125)$ \\
\hline V & $36-50(42)$ & $76-100(89)$ \\
\hline VI & $14-24(19)$ & $52-74(65)$ \\
\hline Somito genital duplo & $29-42(34)$ & $54-76(63)$ \\
\hline \multicolumn{3}{|l|}{ Somitos addominais } \\
\hline 1 & $12-19(16)$ & $46-62(54)$ \\
\hline 11 & $11-16(14)$ & $43-56(51)$ \\
\hline 111 & $10-14(12)$ & $37-47 .(43)$ \\
\hline Ramos caudais & $15-19(18)$ & $16-18(17)$ \\
\hline Setas caudais & $160-200(178)$ & - \\
\hline Sacos ovígeros & $272-272(272)$ & - \\
\hline
\end{tabular}

OBS: Os números entre parênteses se referem às médias 
Tabela 2. Medidas $(\mu \mathrm{m})$ das antênulas e antenas de 10 fềmeas adultas de Gamidactylus bryconis sp. n.

\begin{tabular}{lccc}
\hline & & COMPRIMENTO & LARGURA \\
\hline $\begin{array}{l}\text { Antênula } \\
\text { Antena }\end{array}$ & $86-104(96)$ & $16-22(20)$ \\
Segmento & & & \\
& 1 & $50-73(63)$ & $32-52(40)$ \\
& 2 & $66-80(76)$ & $23-34(28)$ \\
& 3 & $33-44(39)$ & $14-18(16)$ \\
Garra & & $34-48(44)$ & $6-9(8)$ \\
Espinho móvel & $22-42(34)$ & $\ldots$ \\
\hline
\end{tabular}

Tabela 3. Número de espinhos e setas das pernas de 10 fêmeas adultas de Gamidactylus bryconis sp. n.

EXOPÓDITO

\section{Perna}

\begin{tabular}{|c|c|c|}
\hline 1 & $|-0,0-1| \mid-5$, & $0-1,11-5$ \\
\hline II & $1-0,0-1,0,5$ & $0-1,0,2,0-4$ \\
\hline III & $1-0,0-1,0-5$ & $0,1,0-2,0-4$ \\
\hline IV & II-4 & $0-1,0-4$ \\
\hline
\end{tabular}

mesmo tendo hospedeiros täo próximos.

\section{Bibliografia citada}

BOEGER, W. A.; THATCHER, V. E. 1988. Rhinergasilus piranhus gen. et sp $\mathrm{n}$. (Copepoda, Poecilostomatoida, Ergasilidae) from the nasal cavities of piranha cajú, Serrasalmus natteneri, in the Central Amazon. Proc. Helminthol. Soc. Wash., 55(1):87-90.

SANTOS-SILVA, E. N.; ROBERTSON, B. A.; REID, J. L. W.; HARDY, E. R. 1989. Atlas de copépodos planctônicos, Calanoida e Cyclopoida (Crustacea), da Amazônia Brasileira. I. Represa de Curuá-Una, Pará. Rev. Bras. Zool. 6(4):725-758.
SMITHE, F. B. 1974. Naturalist's Color Guide and Supplement. Amer. Mus. Nat. Hist. New York, N.Y. 229p., Colors 1-86.

THATCHER, V. E. 1991. Amazon Fish Parasites. Amazoniana, 11(3/4):263-571.

THATCHER, V. E.; BOEGER, W. A. 1984. The parasitic crustaceans of fishes from the Brazilian Amazon, 13. Gamidactylus jaraquensis gen. et sp. nov. (Copepoda: Poecilostomatoida: Vaigamidae) from the nasal fossae of Semaprochilodus insignis (Schomburgk). Amazoniana, 8(3):421-426.

THATCHER, V. E; ROBERTSON, B. A. 1984. The parasitic crustaceans of fishes from the Brazilian Amazon, 11. Vaigamidae fam. nov. (Copepoda, Poecilostomatoida) with males and females of Vaigamus retrobarbatus gen. et sp. nov. and $V$. 
spinicephalus sp. nov. from plankton. Can. J. Zool, 62:716-729.

VARELLA, A. M. B. 1992. Copépodos (Crustacea) parasitas das fossas nasais de peixes, coletados na região de Rondônia, Brasil. Tese de Doutorado, Instituto de Biociências, UNESP-Rio Claro, São Paulo, 105p. 